Part of Journal of Research of the National Bureau of Standards, Volume 34, May 1945

\title{
METHODS FOR THE EVALUATION OF ANALYTICAL FILTER PAPERS
}

\author{
By B. W. Scribner and W. K. Wilson
}

\begin{abstract}
As part of an investigation of analytical filter papers for the purpose of assisting in the development of standards of quality, improved methods for rate of flow of water, retention of fine precipitates, and determination of ash were developed. The use of complicated apparatus for measuring the rate of flow of water was avoided by applying Darcy's law to the ordinary conical filter. The use of prefiltered water in making the measurement was found necessary for satisfactory reproducibility. As the standard method for determining the ash content of paper is not sufficiently accurate for filter paper, the method was modified in that a tare crucible is used and the test specimen wetted and pressed into a compact wad to permit the use of as small a crucible as possible.

The other tests were bursting strength of wet paper, thickness, weight per unit area, alpha cellulose, copper number, and acidity $(\mathrm{pH})$. The last three named are of importance relative to the purity of the cellulose and the stability of acidwashed papers, as instances of deterioration of the cellulose of such papers have been observed. Action of acid results in brittling of the papers and in the formation of modified forms of cellulose that are soluble in some solutions.
\end{abstract}

\section{CONTENTS}

$\begin{aligned} & \text { Page }\end{aligned}$

I. Introduction

II. Testing methods

1. Retentiveness to fine precipitates

2. Flow of water $\ldots \ldots \ldots 4$

3. Content of ash

4. Bursting strength of wet paper_... _.. 457

5. Thickness, weight, and density

6. Stability _.... 457

\section{INTRODUCTION}

As a part of an investigation at the National Bureau of Standards to establish standards for analytical filter papers, testing procedures for determination of their quality have been developed. It is believed that the various tests are adequate for the evaluation of properties of importance in ordinary analytical work. The tests are being applied to nearly all types and grades of papers, both foreign and domestic, now available in this country, and experience has indicated that the tests are suitable for effecting a standardization of quality for analytical filter papers. When additional testing is completed, the issuance of a report dealing with quality standards is contemplated.

Improved methods were developed for testing for retention of barium sulfate, rate of flow of water through the papers, and content 
of ash. A method was also developed for determination of the bursting strength of wet paper. The following additional testing methods are used. These are standard methods of the Technical Association of the Pulp and Paper Industry (TAPPI), ${ }^{1}$ and the Association's designations of the methods are included.

Thickness ( $\mathrm{T} 411 \mathrm{~m}$ ) and weight in grams per square meter $(\mathrm{T} 410 \mathrm{~m})$. Density is calculated from these values.

Alpha cellulose ( $\mathrm{T} 429 \mathrm{~m}$ ), copper number ( $\mathrm{T} 430 \mathrm{~m}$ ) and acidity as $\mathrm{pH}(\mathrm{T} 435 \mathrm{~m})$. These are related to the purity of the cellulose and the stability of the papers.

Discussions and descriptions of the methods follow.

\section{TESTING METHODS}

\section{RETENTIVENESS TO FINE PRECIPITATES}

The use of a precipitate of barium sulfate is a time-honored practice for testing filter papers for retentiveness to fine precipitates, and it appears to be satisfactory for the purpose. However, it is necessary that the following instructions be followed exactly:

Dissolve $0.55 \mathrm{~g}$ of potassium sulfate in $275 \mathrm{ml}$ of water and add 1.0 $\mathrm{ml}$ of concentrated hydrochloric acid. This solution contains about $0.1 \mathrm{~g}$ of sulfur. Heat to boiling, and while at the boiling temperature, add slowly and with continuous stirring $25 \mathrm{ml}$ of 5-percent barium chloride solution. It is very important to have the specified concentration of acid and to keep the solution at the boiling temperature during the precipitation. Allow to stand without agitation for not less than 2 hours nor more than 6 hours at a temperature below the boiling point and not less than $70^{\circ} \mathrm{C}$. Prepare at least four circles of the filter paper in the usual way for filtration, in 60-degree longstemmed funnels. Stir the precipitate until it is evenly suspended in the liquid and filter approximately $50 \mathrm{ml}$ of the mixture through each circle of paper, collecting the filtrates in 250-ml Erlenmeyer flasks. Swirl the filtrates to bring any sulfate to the center and view from above against a black background for the presence of barium sulfate. The experience at the Bureau is that $0.3 \mathrm{mg}$ or less can be detected in this manner. The paper is considered unsatisfactory if any barium sulfate is seen in the filtrate.

\section{FLOW OF WATER}

A report on the rate of flow of water through filter paper has already been published as a part of this investigation. ${ }^{2}$

Various forms of apparatus were devised by other investigators, but a satisfactory method was evolved by Bogaty and Carson which does not require the use of special, complicated apparatus, and has the advantage of simulating the manner in which filtrations are made. The filter paper is folded in a cone in the usual way, taking care to expel air pockets, and, to obviate any variable that might be introduced by the use of a funnel, the cone is suspended freely in a wire loop. The change in head and filtering area in the use of a cone introduces no complications because, according to Darcy's law, the

\footnotetext{
${ }_{1}$ Copies of the TAPPI methods can be obtained from the Association at 122 East $42 d$ St., New York $17, \mathrm{~N}$. Y.

3 Bogaty, H., and Carson, F. T., Measurement of rate of flow of water through filter paper, J. Research NBS 33, 353 (1944) RP1613.
} 
rate of filtering of water for a given filter paper is proportional to the area of the filter and to the head of water. With this relation as a starting point it was found possible to derive a simple flow constant applicable to a conical filter, which gives the speed of filtering from a measurement of the time required to filter half the volume of water remaining in the cone (starting with any convenient, known volume).

The water-filtration coefficient, $k$, is determined by means of the equation

$$
k=\frac{1.151 \log \frac{v_{1}}{v}}{t-t_{1}}
$$

in which $t-t_{1}$ is the time interval required to decrease the volume of water in the filter cone from $v_{1}$ to $v$. If the measurements are so made that $v=v_{1} / 2$, and $t_{1}$ is taken as zero when the volume in the cone is $v_{1}, k=0.3465 / t$ for any value of $v_{1}$ that may $\mathrm{be}^{\prime \prime}$ chosen. In order to avoid very small numbers, the rate of flow through the filter paper is designated by the coefficient $K$, where $K=10^{4} k=3465 / t$. $K$ is the number of milliliters of water filtered through a square meter of filter paper in 1 second under a head of 1 centimeter of water.

A phenomenon exhibited by filter paper, and other porous materials, is that ordinarily the rate of flow of liquids through them becomes progressively slower with continued flow. As this led to some discrepancies in results obtained for filter paper, considerable study was made to find the cause of it and means of overcoming its effect. No conclusive explanation as to cause was arrived at, and the same appears to be true of explanations offered by other investigators. However, the difficulty was traced to some unknown condition of water used in making the test, as it was found that the change in rate can be almost eliminated by prefiltration of the water. A description of the method follows.

Prepare distilled water by prefiltering it through filter paper, the rate of flow of which is at least as low as that of the paper to be tested. Use two or more filters in series. For each kind of filter paper to be tested a volume of prefiltered water equal to three or four times the volume of the cone will usually be sufficient. If a considerable quantity of water is to be prepared, the filters should be renewed frequently, because they lose their effectiveness with continued flow of water through them.

Carefully fold a filter circle in the usual way to form a 60-degree cone. Place it in a 60-degree funnel and fill it with water prepared in the manner described. Press down the folds to expel all air pockets and to make the three plies smooth and in good contact. Allow about three-fourths of the water to filter through, then pour off the excess. Save the water to wet the next specimen. Remove the empty cone and suspend it freely over a burette by supporting the cone in a loop of wire of such size that it will support the cone at about two-thirds of the distance from its apex.

Use prefiltered water having a temperature of $23^{\circ} \pm 2^{\circ} \mathrm{C}$, and pour into the cone, all at once, a known volume which is approximately two-thirds of the volume of the cone. When one-fifth of the water has filtered through into the burette, start a stopwatch. When half the remaining volume has filtered through, stop the stopwatch and record the time in seconds. Test not less than 10 circles, using 
the same water over and over and adding to it from the prepared supply as found necessary.

The time required to filter half the volume remaining is an inverse measure of the rate of flow, and is substantially the same for any given filter paper, irrespective of the size of the test specimen. Convenient volumes of water initially poured in for filter circles of the sizes most generally used are as follows:

\begin{tabular}{|c|c|}
\hline $\begin{array}{c}\text { Diameter of } \\
\text { circle }\end{array}$ & Initial volume \\
\hline$c m$ & $m l$ \\
11 & 25 \\
9 & 15 \\
7 & 7.5 \\
5.5 & 3.5 \\
\hline
\end{tabular}

\section{CONTENT OF ASH}

The necessity for very accurate determination of ash content, together with the small amount present, requires special modifications of the standard method for testing paper. A large test specimen but as small an ignition crucible as possible are required for good accuracy. These conditions are fulfilled by wetting the filter paper with distilled water, thus permitting compact wadding of the paper for conservation of space. The use of a tare crucible and special precautions in the testing operations are necessary. The details of the method are as follows: ${ }^{3}$

Use 20-ml platinum crucibles with covers. A tare crucible with a cover is carried through all operations exactly the same as the crucibles used for the tests. The tare crucible must have practically the same weight and surface area as the test crucible.

Heat the crucibles and covers in a muffle furnace at approximately $925^{\circ} \mathrm{C}$. Cool in a desiccator and weigh to the nearest $0.1 \mathrm{mg}$. Dry about $6 \mathrm{~g}$ of the paper to constant weight at $100^{\circ}$ to $105^{\circ} \mathrm{C}$ and weigh to the nearest $0.01 \mathrm{~g}$. Wet the paper with distilled water containing not more than 10 parts per million of igneous solids, then roll it into a compact mass with clean fingers. Put the test specimen in a crucible, cover it, and place it in a cold muffle furnace. Start the ignition and after smoking has ceased remove the cover and continue the ignition for 2 hours at $925^{\circ} \mathrm{C}$. Replace the cover, cool and weigh the crucible and ash. The ignition is considered to be complete when the weight of the ash becomes constant after reignition for 30 minutes. All the cooling must be done in a desiccator, and the test crucibles and tare crucible must be allowed to remain on the balance pans the same length of time. The ash must be protected from air currents at all times.

Duplicate determinations shall be made, and they shall agree within 0.003 percent of the weight of the dry paper. Report the average of the values to the nearest $0.01 \mathrm{mg}$ per $11-\mathrm{cm}$ circle of the paper and to the nearest 0.001 percent of the weight of the dry paper.

3 The authors acknowledge the assistance of Herbert F. Launer in the development of this method. 


\section{BURSTING STRENGTH OF WET PAPER}

For determination of the strength of filter paper, resistance of wetted paper to bursting appears to be a logical choice. With the exception that the paper is tested after wetting it, the test is made according to the standard method of the Technical Association of the Pulp and Paper Industry, T $403 \mathrm{~m}$. The paper is clamped in the testing instrument over a circular orifice through which pressure is applied by a compressed fluid until the paper breaks. The pressure is recorded on a maximum-reading gauge and is reported as "points." The procedure is as follows:

Clip together a pack of five circles of the filter paper, and immerse the pack in distilled water at $23^{\circ} \mathrm{C}$ for 5 minutes. Remove the pack, allow it to drain suspended for 10 seconds, then immediately determine the bursting strength. Use enough packs to obtain not less than 10 values. Report the average bursting strength to the nearest onequarter point.

\section{THICKNESS, WEIGHT, AND DENSITY}

The thicknesses of at least 10 separate circles are determined with a dial micrometer, and the average of the values is reported to the nearest 0.0002 in. For determination of weight, a pack of not less than 10 sheets is weighed on a chemical balance, and the weight is reported as grams per square meter to the nearest $0.1 \mathrm{~g}$. The density is calculated from the thickness and weight, as grams per cubic centimeter to the nearest $0.001 \mathrm{~g}$.

\section{STABILITY}

Analytical filter paper as produced in the paper mill has a high degree of cellulosic purity, which is a prime requisite for stability of paper, and every care is taken to keep the paper as free as possible from extraneous materials, as impurities, of course, would interfere with analytical determinations. There should be no question, therefore, of the stability of any of the various types of the papers except those treated with acid to reduce the ash to a minimum or to harden the papers. Acids are very deteriorative to cellulose, and unless the treatment with the strong mineral acids is done very carefully, degradation of the cellulose with resultant embrittlement of the paper fibers may occur. Moreover, degraded cellulose is soluble in some solutions, and therefore may pass into filtrates and cause erroneous analytical results.

The Bureau has found in its studies ${ }^{4}$ of the deterioration of papers that a high content of alpha cellulose and a low copper number are the best criteria of the purity of the cellulose of paper fibers. Alpha is a term used to designate the part of the cellulose that is insoluble in a strong solution of sodium hydroxide under definitely prescribed conditions. It is the pure or unmodified fraction of the cellulose. The copper number designates the amount of copper precipitated by cellulose under certain conditions from a solution of copper sulfate. It represents the amount of unstable modified forms of cellulose present.

\footnotetext{
${ }^{4}$ A. E. Kimberly and B. W. Scribner, Summary report of National Bureau of Standards research on preservation of records, Misc. Pub. NBS M154 (1937).
} 
The Bureau has published extensive data showing the close relation of alpha cellulose, copper number, and acidity as hydrogen ion concentration $(\mathrm{pH})$, to the stability of various other kinds of papers. Some information of this kind has been obtained for filter papers. For example, a double acid-washed filter paper that had become brittle during storage had only 72 percent alpha cellulose, a high copper number of 3.3, and a $\mathrm{pH}$ value of 4.5. Comparable figures for good paper of the same type and grade are alpha cellulose, 96 percent; copper number, 0.4 ; and acidity, $6 \mathrm{pH}$. For good stability, the $\mathrm{pH}$ value of paper should not be below 5 .

In a few other instances noted at the Bureau, papers that had been double acid-washed were so brittle that they burst when used for filtration, and all of these were characterized also by a poor condition of the cellulose. The bursting strength of some of these papers ranged, for dry paper, from 4 to 14 points as compared with around 20 points for underteriorated paper. All the deteriorated papers had been stored under good conditions, and some of them had not been in stock very long.

In view of these experiences with the deteriorated papers, all of which were made by manufacturers of papers that are usually of the highest quality, the use of the recommended tests relating to stability seems desirable for both consumer testing and manufacturing control of acid-washed papers.

WAshington, January 18, 1945. 